\title{
EVIIENCE FOR A SOLAR COMPANION STAR
}

\author{
Richard A. Muller \\ Department of Physics \\ and \\ Lawrence Berkeley Laboratory \\ University of California \\ Berkeley, California 94720
}

\section{DISCLAIMER}

This report was prepared as an account of work sponsored by an agency of the United States Government. Neither the United States Government nor any agency thereof, nor any of their etipioyees, makes any warranty, express or implied, or assumes any legal liability or responsibility for the accuracy, completeness, or usefulness of any information, apparatus, product, or process disclosed, or represents that its use would not infringe privately owned rights. Reference herein to any specific commercial product, process, or service by trade name, trademark, manufacturer, of otherwise does not necessasily constitute or imply its endorsement, recommendation, or lavoring by the United States Government or any agzncy thereof. The views and opinions of authors expressed herein do not necessarily state or reflect those of the United Statcs Government or any agency thereor.

The submicted manuscript has been authored by a contractor of the U.S. government under contract No. DEAC03-76SF00098.

to be published in the Proceedings of the International Astronomical Union Symposium 112, "The Search for Extraterrestria' Life: Recent Developments", June 18-21, 1984, Boston Mass. (D. Reidel Pub. Co, Dordrect Holland) 
EVIDENCE FOR A SOLAR COMPANION STAR

\author{
Richard A. Muller \\ Department of Physics and \\ Lawrence Berkeley Laboratory \\ University of California \\ Berkeley, California 94720
}

\begin{abstract}
Periodicity seen in both the mass extinctions and large impact cratering on earth can be explained if one postulates that the sun has a companion star, orbiting in a moderately eccentric orbit with a major axis of 2.8 light-years. No other explanations that have been suggested are compatible with known facts of physics and astronomy. If the companion is a red dwarf star, the most common kind in the galaxy, then no previous astronomical observations would have found it. A search for red objects with large parallax is now underway at Berkeley, and has a good chance of identifying the star in the near future.
\end{abstract}

\title{
INTRODUCTION
}

In the last few years there have been several major discoveries that have upset the stapdard model of gradual evolution. The first was the discovery by Alvarez et al. that a large extraierrestrial object, a comet or an asteroid, had hit the earth at the time of the mass extinctions at the end of the Cretaceous. The impact would throw a large amount of dust into the upper atmosphere for several months, and species would be killed by the lack of sunlight and the resulting cessation of photosynthesis and sub-freezing temperatures. Despite initial skepticism by some paleontologists and geologists, many of the predictions of the Alvarez model have turned out to be true. These include the discovery of the world-wide nature of the extraterrestrial material ${ }^{2}$ (marked by the presence of iridium), the simultaneous extinction of microscopic plant life $e^{3}$, and recently conclusive evidence that the material in the boundary layer had been subjected to a shock wave 4 . Nevertheless, some paleontologists maintained that it was still not possible for an impact to have caused the extinctions. They angued that the extinctions had been gradual, and took place over a period of $10^{6}-10^{7}$ years. ${ }^{5}$ As I hope to show, I believe that we can now reconcile the concept of gradual extinctions with the impact theory.

The second discovery that shook the standard theory of evolution was made by D. Raup and J. Sepkoski ${ }^{6}$, paleontologists at the University of Chicago. The: had compiled the most complete record of marine extinctions ever assemblec. Is 
this record they found strong evidence that mass extinctions were not rare individual events, but that they occured on a regular schedule: every 26 million years. The last extinction took place about 13 million years ago, so we are halfway between catastrophies. Their paper also met with initial skepticism, in part (I believe) because their results were so revolutionary, and they were so conservative in their claims. I too was initially skeptical, until I studied their mathematics and methods, and duplicated many of their calculations myself. I am now convinced that their discovery will eventually be accepted as one of the standard facts on which evolutionary models must be based.

The data of Raup and Sepkoski is shown in Figure 1. The ordinate shows the percentage of families present in the preceding geologic stage that have disappeared in the subsequent stage. Only species that did not survive to the present are analyzed; thus the extinctions look a little more severe than they actually were. We have used a linear ordinate instead of the logarithmic one used by Raup and Sepkoski. The arrows are drawn with 26 million-year intervals, and they appear to line up with the larger extinctions. Their careful mathematical analysis showed that the a priori probability of such agreement if there was no real effect was about one in a thotiand.

R. P. Feynman once said that the "glory" of physics was thai if something were true, there would be a way of presenting it such that its truth would be obvious. Although I don't claim to have succeeded at presenting the Chicago data in such a way that the effect is "obvious", I would like to show my version anyway in Figure 2. I have taken the data from the Raup and Sepkoski plot (Fig. 1) but for each extinction I have plotted a Gaussian curve, and then superimposed the Gaussians. The area of each Gaussian represents the intensity of the extinction (and is equal to the ordinate for Fig. 1); the width (rms) of each Gaussian is the uncertainty in when the extinction took place, which I took as the greater of the following: uncertainty in the geologic time scale (estimated by Harland et al. ${ }^{\text {) }}$ or half the duration of the stage. I think this plot shows the correspondence found by the mathematical analysis in a way that is more evident.

\section{COMPANION STAR MODEL}

At the time we received the preprint of Raup and Sepkoski (November 1983) we knew that at least two of the mass extinctions in their cycle were associated rith iridium layers, the Cretaceous/Te-tiary and the Eocene/Oligocene. It took nearly two months for us to find a plausible hypothesis that could explain the data and that was consistent with everything else we knew about astronomy and physics. During this period we rejected wany theories, including one that attributed the extinctions to oscillations in the galactic plane. (T'll return to this theory shortly.) Finally, with the help of Marc Davis and Piet fut, we had a model that worked. ${ }^{8}$ Simultaneously, D. Whitmire and A. Jackson ${ }^{9}$ developed an essentially equivalent theory, that arrived at Nature magazine on the same day as ours.

Our model postulates that the sun has a companion star that orbits it with a 26 million-year period. By Kepler's law, this determines the semi-major axis of the ellipse to be $(? 5,000,000\}^{2 / 3}=88,000 \mathrm{~A} . \mathrm{U}$. Changing units and multiplying by 
two, we find that the major axis of the orbit is 2.8 light-years. During perihelion, the companion perturbs the denser regions of the comet cloud, sending a shower of a billion comets towards the inner solar system (which is usually kept relatively clean of comets by the perturbative effects of Jupiter and Saturn). We expect that about two dozen would hit the earth. The dinosaurs had survived many such impacts before they were destroyed by .he very large one at the end of the cretaceous.

From the fact that this companion star has not been identified, we can conclude it must be dimmer than 7 th magnitude (i.e. not in the Yale bright star catalog). To be on the main sequence, the star must then be less than about 0.3 solar masses. To have perturbed the comets tile star must be greater than about 0.05 solar masses. In order to come sufficiently close to the sun to affect the comets, the orbit must have eccentricity between 0.6 and 1.0. (Whitmire and Jackson assumed a lower mass in order to make the star invisible; this forced them to an eccentricity close to 1.0. We saw no reason to assume the star invisible, since most of the stars in the sky have never had their distance from us measured.) These are very common eccentricities in multiple star systems; one expects, for orbits distributed randomly in phase space, that half of them would have eccentricity greater than 0.7 . The mass range suggests the star is a red dwarf, which is the most common stellar type in the Galaxy. Thus there was no need to postulate anything new or exotic.

Piet Hut was able to show that such an orbit would be stable for about $10^{9}$ years against breakup by passing stars and molecular clouds. Galactic tides tend to increase the effective binding of the star to the sun in the direction perpendicular to the galactic plane; they give a restoring force. Hut deduced that if the oscillations of the star are in this direction, then the major axis must be slightly larger than 2.8 light years (to keep the period at $26 \mathrm{Myr}$ ) and so the orbit is slightly less stable to perturbations of passing stars. The stability of the orbit is sufficientiy lons to account for the regularity in the extinctions, but it also implies that the sompanion star could not have been in this orbit since the formation of the earth. Since capture is very improbable, the most likely scenario is that the companion was once more tightly bound, and is slowly being evaporated by passing stars. It is conceivable that crater records will prove this speculation, since they hold the record of the periodicity and intensity of past comet showers. It is possible, for example, that the end of the late great bombardment of the moon that ended 3.9 billion years ago came about when the companion star was scattered from a close-in orbit to one much further out. It is interesting to note that the first evidence for life on earth in isotopic records occurs just after the end of this bombardment.

Another suggestion has been made, that the extinctions pere triggered by the passages of the solar system through the galactic plane 10 , The times of the galactic plane crossings, as presented by Rampino and Stothers $11^{1}$, are plotted against the mass extinctions in Figure 3. Not only is there no obvious correlation, but in the period when the times are most accurately determined (the recent past), the phase appears to miss by $180^{\circ}$. In other words, although we are halfway between extinctions, the solar system is in the galactic plane now. Further computer simulations confirm the visual impression of a poor fit. We generated 10,000 sets of "random data" in which we picked 10 dates randomly between 0 and 
250 Myr ago; $46 \%$ of these random sets had a smaller RMS deviation from the extinction dates than had the galactic plane crossings. In contrast an exact 26 Myr period fits the extinctions better than $99.5 \%$ of the random data sets. Rampino and Stothers mistakenly claim in their paper that the probability of random data doing as well as the galactic plane crossing is not $46 \%$ as we found, but $0.4 \%$ ! Their incorrect number is due to a simple mathematical mistake: in calculating the a priori probability of agreement, they used the value one would get for agreement between two sets of unordered numbers, but the sets they compared (dates of extinctions and dates of galactic plane crossings) were both ordered (i.e. they both increase monotonically).

\section{EARTH CRATERING PERIODICITY}

After our companion model was submitted to Nature, Walter Alvarez realized that the comet shower model made another implicit prediction concerning the dates of impact craters on the earth. Although the probability of finding a crater from an earth impact may be $10 \%-25 \%$ (since many impact regions, such as the ocean floors, have not yet been studied), the comet shower model implied multiple impacts, so that the probability of finding at least one crater from a given shower should be high. To our delight, in a compilation of crater ages. ${ }^{12}$ we found that they had an apparent $28 \mathrm{Myr}$ peripdicity agreeing (within errors) with the frequency and phase of the extinctions. 3 Most of the effect was coming from the larger craters (greater than $10 \mathrm{~km}$ diameter); the ages of these craters are plotted in Figure 4, along with arrows indicating the $28 \mathrm{Myr}$ periodicity. Fourier analysis and Monte-Carlo simulations showed ${ }^{2}$ that the error associated with the period was about $\pm 1 \mathrm{Myr}$, and the error associated with the time of the most recent event was about \pm 2 Myr. The period when a nominal 26 Myr and a $28 \mathrm{Myr}$ period would get out of phase, 150-200 Myr ago, is the time when the paleontological data is reakest (see Fig. 1) and when the ages of the geological stages are most tuisertain. The analysis proved to be rather robust against changas in the data set, including the addition or elimination of a few craters, or changes in the minimum crater diameter included.

\section{PREDICTIONS}

This new model of the mass extinctions makes several new predictions. The obvious one is the existence of the companion star. (If it is found, we suggested it be called "Nemesis".) I will discuss our ongoing search for this star later. A nother important prediction is that all the extinctions seen by Raup and Sepkoski are associated with comet impacts, and should have associated iridium layers. Subsequent to our work, such an iridium layer was found ${ }^{14}$ at one of these layers, the Peimian/Triassic. Fie also predict that some, if not all, of the mass extinctions will be associated with multiple impacts. This suggests that we look for multiple iridium layers. The best that one can say now is that there is no good evidence against multiple irjdium layers; the published data are consistent with the existence of several peaks. A new iridium detection apparatus now under construction in the Aivarez group, based on coincident $317 \mathrm{keV}$ and $468 \mathrm{keV}$ gamwa rays ffgm the decay of $\mathrm{Ir}-102$, Fil! he capab!e of measuring the levels as low as $5 \times 10^{-12}$ without prior chernical purification, and should be able to test both predictions. 


\section{EXTENDED EXTINCTIONS}

The duration of the comet shower depends on the eccentricity of the companion's orbit. A rough estimate of the duration of the shower can be made by calculating the strength of the perturbation (proportional to $1 / r^{3}$ ) as a function of time; this is shown in Figure 5 for eccentricities of 0.6 to 0.8 . If we arbitrarily define the duration of the shower to be the width of the peaks in the curve (FWHM), then the duration of the shower is plotted in Fig. 6. For reasonable ranges of eccentricity, 0.6 to 0.9 , the duration of the shower (full-width at halfmax) is 100,000 years to $2,000,000$ years. Very high (and relatively unstable) eccentricities are required to have very short showers. Thus we expect a typical shower, with perhaps 10 impacts spread over I Myr, with an intervals averaging 50,000 years between impacts.

An interesting consequence of the comet shower model is that we would not necessarily expect all species to die out simultaneously during a shower. Some species could be destroyed by an early comet impact, while others make it through, only to be killed by a later and larger impact. The claim of some paleontologisis that the extinctions were not sudden but spread over I Myr or more is no longer in obvious contradiction to the impact model. The shower model does predict that, given sufficient time resolution, each catastrophe could be resolved inio a short series of abrupt events.

\section{EVOLUTION}

If all this is right, then evolution on the earth has proceeded through a vastly different history than we had previously supposed. Since Darwin, we have assumed that the main driving force of evolution is competition between species. Our new model says that such evolution takes place only during the relatively quiet period between comet showers. Every 26 million years or so a new mechanism comes into play; the earth is hit by a world-wide disaster. Without such a catastrophe, mammals might never have wrested the earth from the dinosaurs. We don't know how important this mechanism is for evolution as we know it, but it is possible that it is essential. It may prevent species "stagnation" by killing most of the dominant species and opening new ecological niches for previously suppressed species to occupy. It may play a role in evolution similar to that played by "death" in our everyday lives, making possible the introduction of "young blood". Without death to open some new niches, for example, I could never have obtained tenure at my university.

Since I am a physicist, I have no credentials to lose in the theory of evolution. So I have been able to speculate freely. Future speculation on evolution I should leave to the real experts. Let me return to physics.

\section{OTHER PHYSICS AND ASTROPHYSICS}

If the sun does have a companion star, then there is a new mechanism in our solar s;stem which may help us to explain previously mysterious phenomena. Was the late freat bombardment of the moon from a constant comet shower, triggered 
by a small orbit for the companion? Did this bombardment prevent life from forming, or just obliterate its record? Could the comet showers help explain other phenomena in the solar system such as the existence of the Apollo objects and planetary rings (especially the short-lived rings, such as those on Jupiter)? Perhaps the presence of a companion star will help us to understand the existence of the comets themselves. Did the showers play any role on earth geology, e.g. triggering volcanos or earthquakes? Did the showers contribute significant material to planetary atmospheres? If material is thrown into solar orbit after an earth impact, there may be a recurrent shower every year as the orbits of the earth and the debris intersect at the same place; could this mechanism deliver an iridium layer without blackening the sky and causing extinctions? Or would it just cause multiple-Tunguska type disasters for many decades.

\section{THE BERKELEY SEARCH FOR THE COMFANION STAR}

We know a lot about the hypothesized companion star. It must be between 2.5 and 2.8 light-years away (depending on eccentricity). Its mass is less than 0.3 and probably more than 0.05 solar masses, so it is very likely a red dwarf. Its proper motion and radially velocity are virtually zero, so it never would have been detected by other searches that have been made for nearby stars.

Unfortunately, we don't know what direction to look. Perturbations from this star now should be smaller than those from alpha-centauri and the galactic gravitational gradient. Fortunately, my group at Berkeley over the past four years has been developing an instrument that turned out to be ideally suited to a search for the companion. Using a small automated telescope (either the $30^{\text {n }}$ at Leuschner or the $36^{\prime \prime}$ at the Monterey Institute for Research in Astronomy) we can survey a large number of objects in a shcrt period, and obtain electronic photographics with a CCD (charge-coupled device) canera. We are presently taking such photographs of 5000 red stars in the northern hemisphere. We will return in 3 to 6 months to take a second set of photographs. In each photograph we measure (in a computer) the distance between the red star and other "fiducial" stars in the same field. If the apparent position of the star changes, then the distances in the second set of photographs will be different.

The $30^{n}$ Leuschner telescope is operated remotely by our PDP-11/44 computer, linked via telephone line to an IBM-PC at the observatory. An observer at the telescope monitors the operation, but virtually everything (movement of telescope and dome, opening of a shutter, recording of image, and readout to a video tafe recorder) is automated. We average about 75 star fields per hour in this mode. Our survey of the 5000 red stars should be complete by the end of 1984. A survey of the southern sky could be done with an automated telescope, or with emulsions and a Schmidt camera, and we are currently investigating both of these possibilities.

If the star is not found among the red stars, then it is possibiy a brown dwarf, a dim star with mass probably less than 0.07 solar masses. Good candidates would be stars bright in the IRAS catalogue which are dim in the visible. Unfortnately IRAS did nrt have sufficient angular accuracy (20 arc sec) to be able to see the experced 3 are sec parallax. When a list of IRAS candidates is 
The submitted manuscript has been authored by a contractor of the

U.S. Government under contract No. DEAC03-76SF00098.

\section{REFERENCES}

1. L. W. Alvarez, W. Alvarcz, F. Asaro, H. Michel, Science 208, 1095-1108 (1980).

2. L.W. Alvarez, Proc. Natl. Acad. Sci. USA, 80, pp.627-642 (January 1983).

3. C.J. Orth, J. S. Gilmore, J.D. Knight, C.L. Pillmore, R.H. Tschudy, J.E. Fassett, Science 214, 1341-1343 (1981).

4. B. F. Bohor, E. E. Foord, P. J. Modreski, D. M. Triplehorn, Science 224, 867869 (1984).

5. S. M. Stanley, "Mass Extinctions in the Oceans", Scientific American 250, p. 64-72 (June 1984).

6. D. Raup and J. J. Sepkoski, Proc. Nat. Acad. Sci. 81, \$01-805 (1984).

7. W. B. Harland, et al., A Geologic Time Scale, (Cambridge University Press, 1982).

8. M. Davis, P. H•t, and R. A. Muller, Nature 308, 715-717 (1984).

9. D. Whitmire, A. Jackson, Nature 308, 713-715 (1984).

10. M. R. Rampino and R. B. Stothers, Nature 308, 709-712 (1984).

11. R. D. Schwartz and P. B. James, Nature $\underline{308}, 712-713$ (1984).

12. R. A. F. Grieve, Geol. Soc. Am. Spec. Pap. 190, 25-37 (1982).

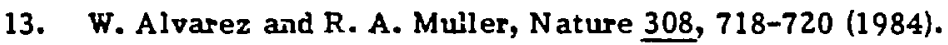

14. X. Dao-Yi, M. Shu-Lan, C. Zhi-F'ang, M. Xuo-Ying, S. Yi-Ying, Z. Oin-Wen, Y. Zheng-Zhong, "Abundance Variation of Iridium and Trace Elements at the Permian-Triassic Boundary at Shangsi, Guangyuan, Sichuan, China", to be published; also, S. yi-Ying et al., "The Discovery of Iridiun Anomaly in the Permian-Triassic Boundary Clay in Changxing, Zhejiang, China and its significance", to be published.

15. J. T. Kare, C. R. Pennypacker, R. A. Muller, T. S. Mast, F. S. Crawford, and M. S. Burns, Lawrence Berkeley Laboratory report LBL-13317, also in "Supernovae: A Survey of Current Research", M. J. Rees and R. J. Stoneham, eds, p. 325-339 (D. Reidel, Dordrect 1981). 


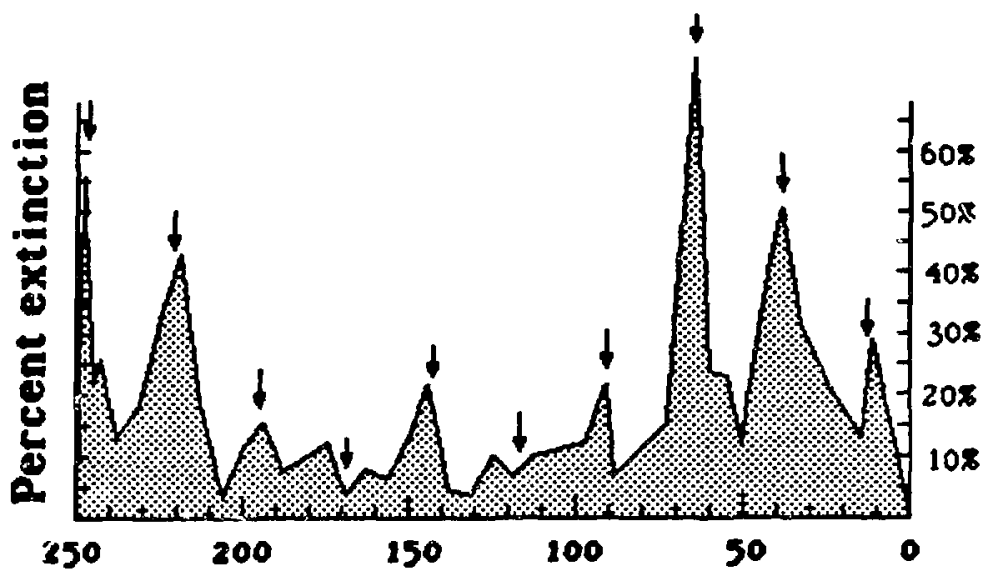

Million years before present

Fig.1. Family extinctions in the oceans, adapted from $R$ aup and Sefkoski ${ }^{6}$. The percentage of families present in a preceding stage and extinct in the following is plotted at the stage boundary. The vertical scale is linear, not logarithmic as in the original paper. Species still alive at the present were excluded from the data set. The arrows indicate the $26 \mathrm{Mgr}$ periodicity.

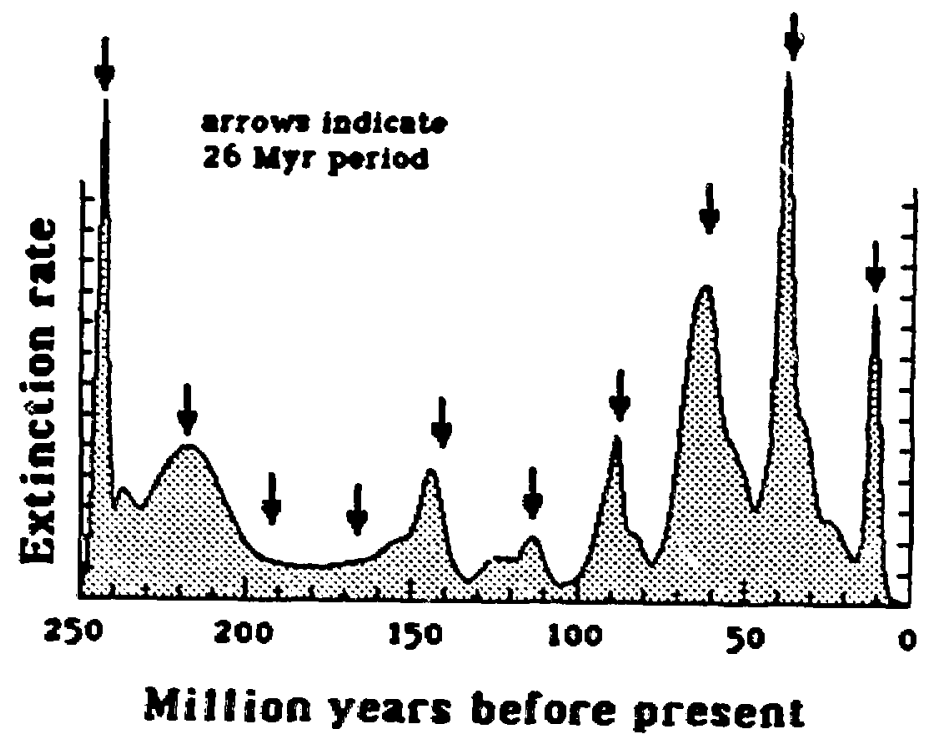

Fig.2. Familiy extinctions versus time; tame data as Figare 1 but plotted differestly. At each boundary a Gaussian curve was plotted, with width equal to the uncertainty in the tine of the extinction, and area equal to the percentage of fawilies that became extinct. The Gaussians were added to si ae the curre shows. The arrows indisate the $26 \mathrm{Myr}$ periodicicy. 


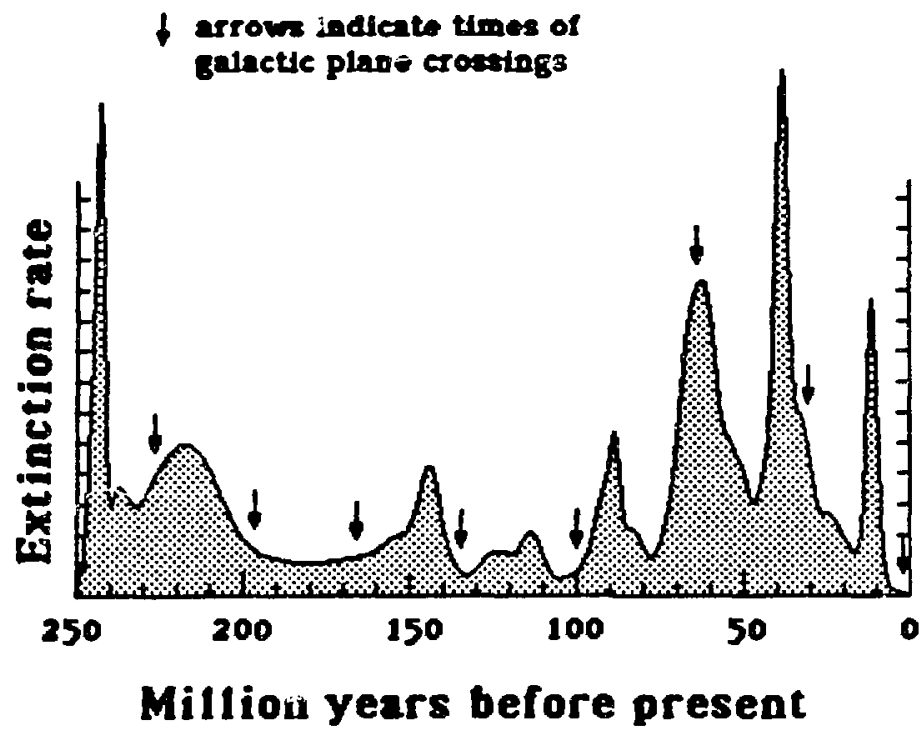

F.g.3. Galactic plane crossings: same curve as Figure 2 but now the arrows indicate the times when the solar system passed through the galactic plane. The correlation between the extinctions and the crossings is no better than for totally random data.

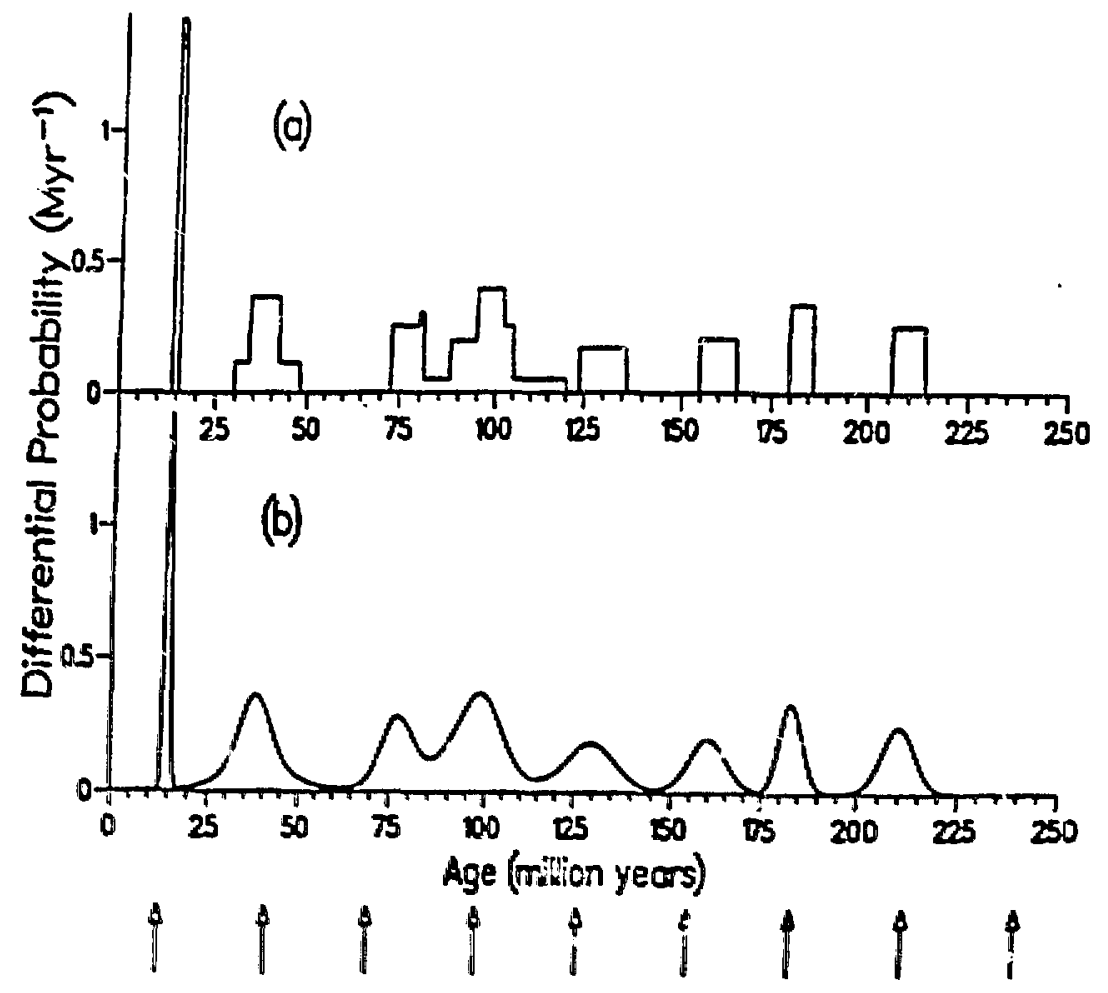

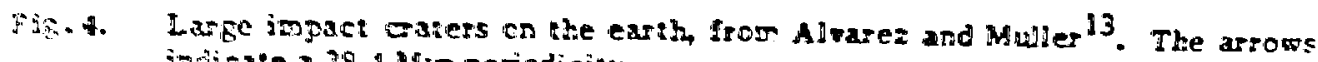
indicate a 2 . A My pericdiciny. 


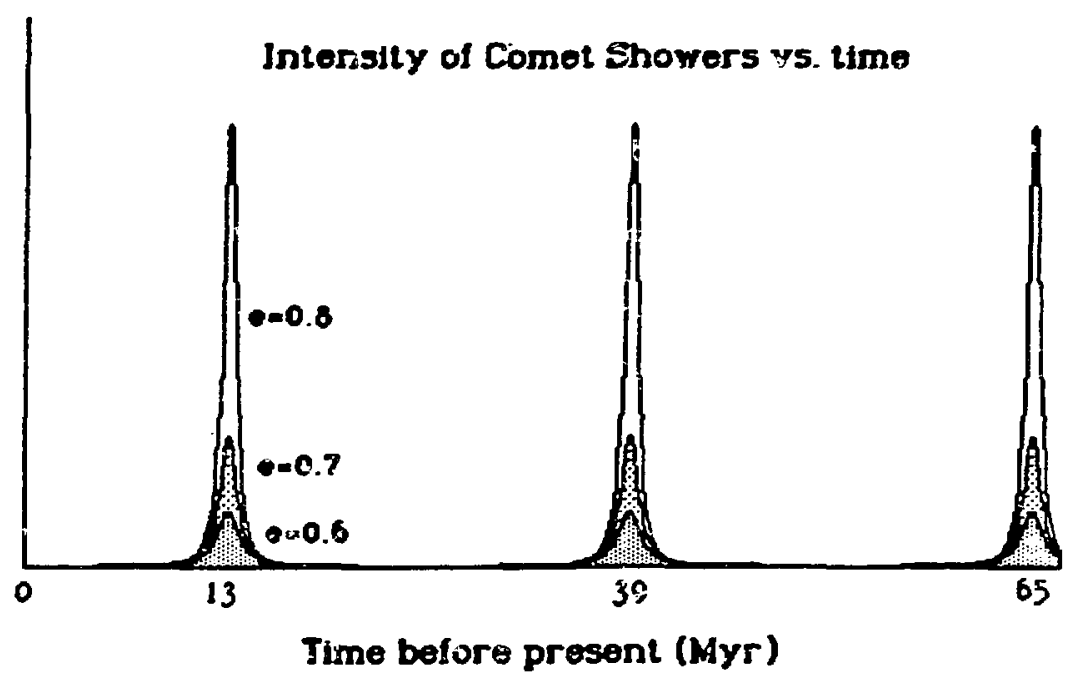
Fig. 5. Intensity of the companion star perturbation $1 / r^{3}$ vs. time, for different
eccentricities of the arbit.

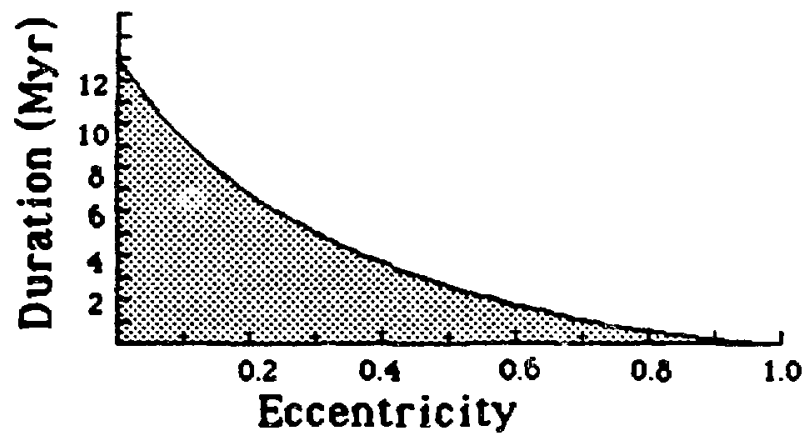

Fig. 6. Duration of comet showers, as a function of eccentricity. The full-widtihalf-max of the perturbation curve (Figure 5) is plotted vs. eccentricity. 
available, we can study the parallax in the visible using our automated telescope. If there is no visible component, then we may have to attempt parallax in ihe infrared from the ground, or wait until the Space Telescope is available.

\section{SUMMARY}

I will try to distinguish herc between those facts which I feel have been established, and those theories which are speculative. I feel that the following facts have been established by the data:

\section{established}

1. There is a periodicity in evolution on the earth; mass extinctions occur with a regular $26 \mathrm{Myr}$ period.

2. At least three of these extinctions occured simultaneously with the impact cf a comet or astercid on the earth.

3. Large impact cratering on the earth occurs with a $28 \pm 1 \mathrm{Myr}$ period, equal within errors to the period seen in the mass extinctions, with the same phase.

The following conclusions, while not as firmly established as the above facts, followly relatively directly from them without need for a model. They do not, for example, depend on the existence of a solar companion star.

\section{very likely true}

1. All of the periodic extinctions seen by $\bar{R}$ aup and Sepkoski are caused by showers of comets or asteroids. (That "showers" are required is a consequence of Poisson statistics, and the small likelihood that individual objects will bit the earth.)

2. The next shower is due in about 13 million years.

3. These showers have played an important role in evolution that had not previously been recognized.

The following conclusions are not as firmly established as the two above, and are based primarily on the absence of competing models that are consistent with the known astrophysical and paleontological data.

1. The sun has a companion star, orbiting with a moderately eccentric orbit with a major axis of 2.8 light-years.

2. The duration of a comet shower should be berween a few hundred thousand years and a few million years.

If the comparion star does exist and is not an exotic object, then dedicated searches such as ours are likely to find it in the next few years. 
This report was done with suppor from the Department of Energy. Any conclusions or opinions expressed in this report represent solely those of the author(s) and not necessarily those of The Regents of the University of California, the Lawrence Berkeley Laboratory or the Department of Energy.

Reference to a company or product name does not imply approval or recommendation of the product by the University of Califomia or the U.S. Department of Energy to the exclusion of others that may be suitable. 